\title{
Ultrafast Demagnetization Dominates Fluence Dependence of Magnetic Scattering at Co $M$ Edges
}

\author{
Michael Schneider $\odot,{ }^{1}$ Bastian Pfau $\odot,{ }^{1}$ Christian M. Günther $\odot,{ }^{2,3}$ Clemens von Korff Schmising $\odot,{ }^{1}$ David Weder, \\ Jan Geilhufe $\odot,{ }^{1}$ Jonathan Perron, ${ }^{4}$ Flavio Capotondi $\odot,{ }^{5}$ Emanuele Pedersoli $\odot,{ }^{5}$ Michele Manfredda, ${ }^{5}$ \\ Martin Hennecke®, ${ }^{1}$ Boris Vodungbo, ${ }^{4}$ Jan Lüning, ${ }^{6}$ and Stefan Eisebitt ${ }^{1,2}$ \\ ${ }^{1}$ Max Born Institute for Nonlinear Optics and Short Pulse Spectroscopy, Max-Born-Straße 2A, 12489 Berlin, Germany \\ ${ }^{2}$ Technische Universität Berlin, Institut für Optik und Atomare Physik, Straße des 17. Juni 135, 10623 Berlin, Germany \\ ${ }^{3}$ Technische Universität Berlin, Zentraleinrichtung Elektronenmikroskopie (ZELMI), Straße des 17. Juni 135, 10623 Berlin, Germany \\ ${ }^{4}$ Sorbonne Université, CNRS, Laboratoire de Chimie Physique-Matière et Rayonnement, LCPMR, 75005 Paris, France \\ ${ }^{5}$ Elettra Sincrotrone Trieste S.C.p.A., Strada Statale 14, km 163.5, 34149 Basovizza, TS, Italy \\ ${ }^{6}$ Helmholtz-Zentrum Berlin für Materialien und Energie, Hahn-Meitner-Platz 1, 14109 Berlin, Germany
}

(Received 13 August 2019; revised 27 March 2020; accepted 7 August 2020; published 15 September 2020)

\begin{abstract}
We systematically study the fluence dependence of the resonant scattering cross-section from magnetic domains in Co/Pd-based multilayers. Samples are probed with single extreme ultraviolet (XUV) pulses of femtosecond duration tuned to the Co $M_{3,2}$ absorption resonances using the FERMI@Elettra free-electron laser. We report quantitative data over 3 orders of magnitude in fluence, covering $16 \mathrm{~mJ} / \mathrm{cm}^{2} /$ pulse to $10000 \mathrm{~mJ} / \mathrm{cm}^{2} /$ pulse with pulse lengths of $70 \mathrm{fs}$ and $120 \mathrm{fs}$. A progressive quenching of the diffraction cross-section with fluence is observed. Compression of the same pulse energy into a shorter pulseimplying an increased XUV peak electric field-results in a reduced quenching of the resonant diffraction at the Co $M_{3,2}$ edge. We conclude that the quenching effect observed for resonant scattering involving the short-lived Co $3 \mathrm{p}$ core vacancies is noncoherent in nature. This finding is in contrast to previous reports investigating resonant scattering involving the longer-lived Co $2 \mathrm{p}$ states, where stimulated emission has been found to be important. A phenomenological model based on XUV-induced ultrafast demagnetization is able to reproduce our entire set of experimental data and is found to be consistent with independent magneto-optical measurements of the demagnetization dynamics on the same samples.
\end{abstract}

DOI: 10.1103/PhysRevLett.125.127201

With the advent of femtosecond, high-brightness pulses of XUV and x-ray radiation from free-electron laser (FEL) sources, the search for and potential use of coherent lightmatter interaction in this spectral range has intensified. Wave mixing was demonstrated [1,2], and gain, as well as lasing, in the soft $\mathrm{x}$-ray regime were reported [3,4]. Utilizing resonant magnetic scattering in cobalt-based magnetic materials, nonlinear cross-sections at absorption edges exhibiting $\mathrm{x}$-ray magnetic circular dichroism (XMCD) were observed, both at the Co $L_{3}$ edge at about $778 \mathrm{eV}$ photon energy [5-8], as well as at the shallower $M_{3,2}$ edges at about $60 \mathrm{eV}$ photon energy [9]. For processes in resonance with the Co $L_{3}$ edge and fluences per pulse covering the range from $10 \mathrm{~mJ} / \mathrm{cm}^{2}$ to $1600 \mathrm{~mJ} / \mathrm{cm}^{2}$, stimulated emission was argued to be the main mechanism for the nonlinearity observed in the light-matter interaction

Published by the American Physical Society under the terms of the Creative Commons Attribution 4.0 International license. Further distribution of this work must maintain attribution to the author(s) and the published article's title, journal citation, and DOI.
[6-8]. X-ray-induced ultrafast demagnetization was found to be of relevance only for pulse durations significantly longer than $100 \mathrm{fs}$, given moderate fluences of up to $30 \mathrm{~mJ} / \mathrm{cm}^{2}$ [5]. Recently, this mechanism gained renewed attention, even for x-ray pulse durations of only $40 \mathrm{fs}$, i.e., significantly below the typical demagnetization time constants of around 100-300 fs [10]. In the case of the Co $M_{3,2}$ edges, an almost complete loss of resonant scattering signal was reported for a fluence of $5000 \mathrm{~mJ} / \mathrm{cm}^{2}$ in a $100 \mathrm{fs}$ pulse. Here, the explanation focused on the ionization of the sample and the associated change of resonant transition energies during the pulse [9].

Given these observations and the different mechanisms proposed, the total fluence dependence observed is likely to be a combination of competing processes. Which of the different mechanisms dominates will depend on experimental parameters such as the pulse duration, coherence time, and fluence distribution, as well as material-dependent properties like the demagnetization time constant or the respective core-hole lifetime. So far, neither detailed data over an extended fluence range and different pulse lengths nor satisfactory quantitative models are available for the $M_{3,2}$ edges of the $3 \mathrm{~d}$ transition metals. Here, we 
(a)

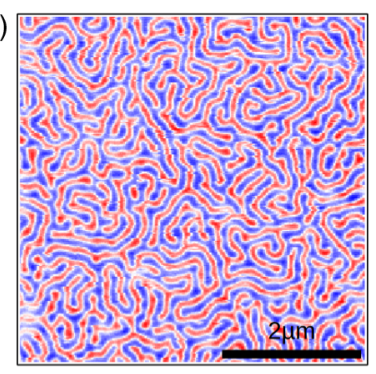

(b)
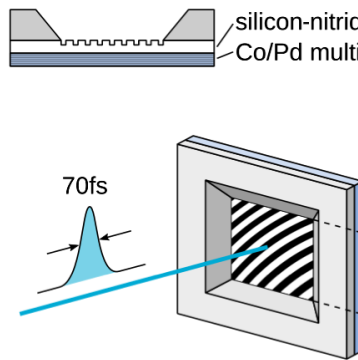

silicon-nitride grating Co/Pd multilayer

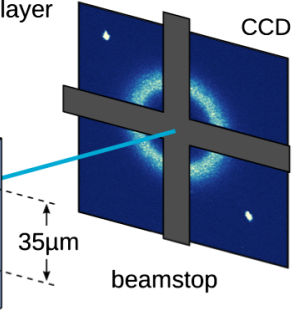

(c)

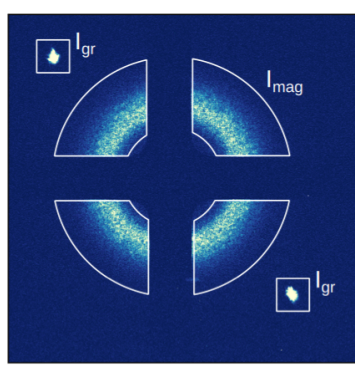

FIG. 1. Sample layout and experiment design. (a) Magnetic force microscopy image of a $5 \mu \mathrm{m} \times 5 \mu \mathrm{m}$ sample area. Blue and red colors indicate opposite magnetization directions perpendicular to the sample plane. (b) Schematic cross-section of the structured sample membrane and sketch of the experiment. The cross-section shows the etched silicon-nitride membrane with the artificial grating structure and the magnetic multilayer. The focused FEL pulse (blue line) with a pulse length of 70 fs or 120 fs first hits the silicon-nitride membrane with the artificial grating structure. A CCD detector $50 \mathrm{~mm}$ downstream of the sample records the XMCD small-angle scattering (SAS) signal. The cross-shaped beamstop protects the detector from the intense directly transmitted light. (c) Representative XMCD-SAS pattern. $I_{\mathrm{mag}}$ and $I_{\mathrm{gr}}$ denote the SAS contributions of the magnetic domain pattern and the milled grating, respectively. White outlines mark the regions in which the respective signals are integrated.

report quantitative data on the scattering cross-section from thin magnetic multilayers with perpendicular anisotropy obtained at the Diffraction and Projection Imaging endstation of the FERMI@Elettra FEL source $[11,12]$. These data cover the incident fluence range of $16 \mathrm{~mJ} / \mathrm{cm}^{2}$ to $10000 \mathrm{~mJ} / \mathrm{cm}^{2}$ per XUV pulse with pulse durations of $(70 \pm 20)$ fs and $(120 \pm 20)$ fs FWHM.

Magnetic multilayers with a nominal composition $\mathrm{Al}(10) / \operatorname{Pd}(2) /[\mathrm{Co}(0.4) / \operatorname{Pd}(0.2)]_{30} / \mathrm{Al}(3)$ (thickness values in $\mathrm{nm}$ ), in a labyrinth state of ferromagnetic domains with alternating magnetization (anti)perpendicular to the substrate surface, are employed as samples. Their domain pattern is prepared to be isotropic in the plane of the film, with an in-plane correlation period of about $200 \mathrm{~nm}$ [Fig. 1(a)]. The thin films are sputtered on $30 \mathrm{~nm}$ thick silicon-nitride membranes with a window size of $35 \mu \mathrm{m} \times 35 \mu \mathrm{m}$. The incident, linearly polarized XUV radiation is tuned to a center wavelength of $20.8 \mathrm{~nm}$ and thus in resonance with core-to-valence transitions of $3 \mathrm{p}_{3 / 2,1 / 2}$ core electrons. XMCD contrast at the Co $M_{3,2}$ absorption edges gives rise to SAS due to the different absorption cross-sections in oppositely magnetized domains. Since the FEL beam footprint is large compared to the domain size, the net magnetization within the probing area is zero, and the SAS intensity is proportional to the square of the local domain magnetization $M$ [13]. As sketched in Fig. 1(b), we detect the scattered intensity on a downstream 2D detector via integration over a suitable region of interest, marked in the scattering pattern in Fig. 1(c).

Curved grating monitors covering the entire membrane area are prepared on each membrane by focused-ion-beam milling. This is done before the sputter deposition of the magnetic layer in order to avoid ion-induced damage. To allow for alignment in the XUV beamline via a long-range microscope, the samples are oriented with the structured silicon-nitride membrane side toward the incident beam.
The shallow topographic gratings generate a scattering signal that is well separated from the XMCD-SAS on the same detector $\left[I_{\text {gr }}, I_{\text {mag }}\right.$ in Fig. $\left.1(\mathrm{c})\right]$. Thus, the integrated gratings allow for a shot-by-shot normalization to the fluence actually incident on each membrane [14]. This is especially important given the complex internal structure of the spatial fluence distribution in the sample plane [15], as well as possible shot-to-shot fluctuations of the beam pointing on a micrometer scale [21]. Additionally, we obtain the detailed spatial fluence distribution by matching extrapolated wave-front sensor measurements [22] with single-shot ablation craters in the sample substrate, recorded regularly to monitor any possible drift of the intensity distribution in the sample plane on the $10 \mathrm{~min}$ timescale [15]. Thus, XUV intensity and pointing variations can be quantitatively accounted for, in addition to any drift on longer timescales. In sum, this allows for a very precise assessment of the incident fluence distribution, which is key for a fluence-dependent measurement. A gas monitor detector measures the integral pulse energy of all individual FEL shots.

After aligning a sample membrane relative to the XUV focus, a diffraction pattern at low fluence $[(16 \pm 4) \mathrm{mJ} /$ $\mathrm{cm}^{2} /$ pulse] is first recorded for normalization purposes. Then, we record a destructive single-shot diffraction pattern from the same membrane at the selected fluence. The initial low-fluence calibration measurement provides a baseline of the magnetic scattering from the particular domain sample and the scattering efficiency of the absorption grating. This allows one to correct for fabrication-related differences in the scattering efficiency of the magnetic layer and the absorption grating [15].

The signal of interest is the relative XMCD-SAS diffraction cross-section, defined as the intensity ratio of magnetic (subscript "mag") and grating ("gr") diffraction in the single shot (superscript "high") relative to the low-fluence characterization ("low"): $S=\left(I_{\text {mag }}^{\text {high }} / I_{\text {gr }}^{\text {high }}\right) /\left(I_{\text {mag }}^{\text {low }} / I_{\text {gr }}^{\text {low }}\right)$. This 

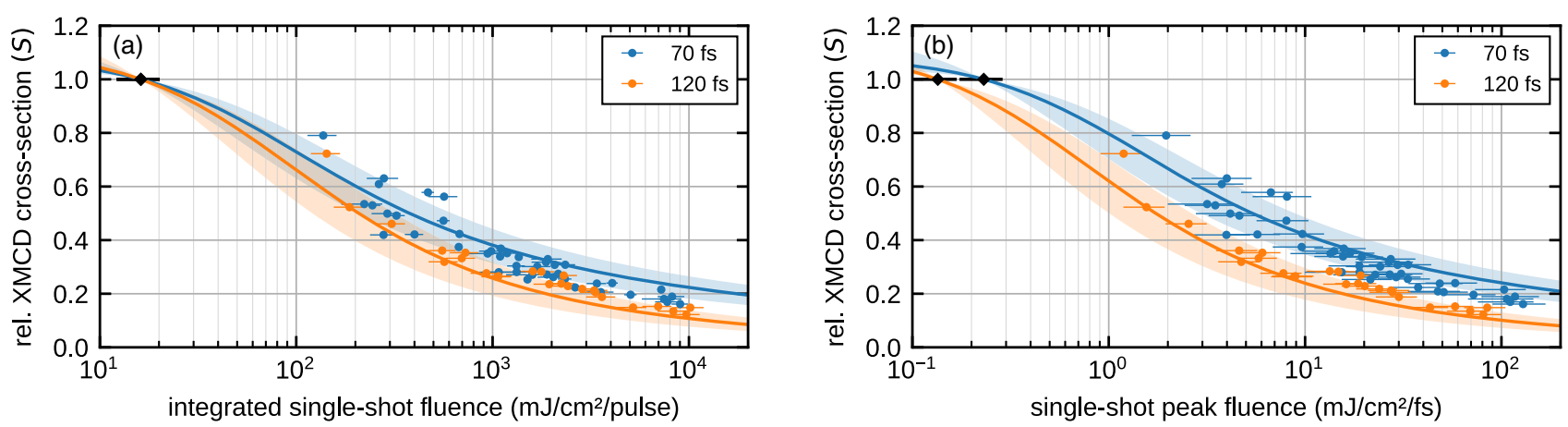

FIG. 2. Fluence dependence of the relative XMCD-SAS cross-section $S$ for two different X-ray pulse lengths (circles), shown as a function of the single-shot pulse energy (a) and normalized per unit time within the pulse, i.e., divided by the pulse duration (FWHM) (b) [15]. The black diamond symbols indicate the characterization fluence of $(16 \pm 4) \mathrm{mJ} / \mathrm{cm}^{2} /$ pulse used in the experiments. Solid lines show the results of a simulation taking only ultrafast demagnetization during the pulse into account. The shaded areas mark the uncertainty of this model, which is predominantly given by the confidence with which the FEL pulse lengths are calculated from machine parameters [15].

ratio represents the relative fluence-dependent change of the XMCD-SAS diffraction cross-section with respect to the low-fluence characterization. Its value is entirely self-contained in two scattering patterns recorded consecutively on the same detector, dispensing the need for any additional scaling, e.g., normalizing by the gas monitor detector or the maximum scattering signal. In Fig. 2, we show the relative diffraction cross-section $S$ for FEL pulse lengths of $(70 \pm 20 \mathrm{fs})$ and $(120 \pm 20)$ fs as a function of the pulse fluence [Fig. 2(a)] and the peak fluence [i.e., divided by the respective pulse duration, Fig. 2(b)] obtained from 70 individual sample membranes. We observe a steady decrease of the XMCD-SAS cross-section, which can now be analyzed quantitatively, comparing different pulse durations.

The most important observation that can be made on the basis of the experimental data alone is this: For a constant fluence on the sample, a shorter pulse duration does not lead to a stronger reduction of $S$. This observation alone is incompatible with a coherent mechanism such as stimulated emission, as the same pulse fluence administered in a shorter pulse implies a higher instantaneous strength of the electric field throughout the duration of the pulse. Consequently, a coherent field-driven process would generate a stronger effect when the same pulse energy is compressed into a shorter pulse for unchanged focusing conditions [15]. On this basis alone, we can rule out stimulated emission as the dominant cause for the observed fluence-dependent quenching of $S$.

To obtain additional quantitative insight, we model the fluence-dependent behavior observed experimentally on the basis of XUV-induced demagnetization during the pulse. This implies that energy deposited into the sample by the traversing XUV radiation triggers a demagnetization comparable to optical, infrared, or indirect excitation by hot electrons [23-25]. While the FEL pulse traversing through the sample continuously and simultaneously excites and probes the sample, it is apparent from the modeling how the earlier parts of the XUV pulse lead to a substantial demagnetization. This is probed predominantly via the later parts of the pulse, resulting in a reduction in the XMCD-SAS cross-section [15]. In agreement with the experimental observation, it is expected from this model that even at very high FEL fluences, a residual scattering is present, originating from the probing of the initially fully magnetized system by the very early parts of the pulse. Naturally, this self-induced demagnetization will be stronger for longer pulse durations, matching the experimentally observed behavior: the data for the longer $(120 \pm 20)$ fs pulses consistently show a lower XMCD-SAS cross-section compared to the $(70 \pm 20)$ fs data at similar fluences [15]. A similar mechanism has been discussed for the optical regime [26] and was previously used to explain fluence dependence in nondestructive experiments at the Co $L_{3}$ edge with fluences ranging up to $30 \mathrm{~mJ} / \mathrm{cm}^{2} /$ pulse and pulse lengths of $80 \mathrm{fs}$ and $360 \mathrm{fs}[5,15]$. We fit our phenomenological model to the collective experimental data with only two global fit parameters: the single exponential demagnetization time constant $\left(\tau_{m}\right)$ and the saturation energy density $\left(F_{\text {sat }}^{\text {absorb }}\right.$ ) of the XUV-induced ultrafast demagnetization. The latter parameter is the amount of energy absorbed per sample volume at which the demagnetization eventually reaches $100 \%$ (for $t \rightarrow \infty$, ignoring remagnetization). Up to this point, the magnetization quenching is directly proportional to the energy absorbed with a slope $1 / F_{\text {sat }}^{\text {absorb }}$. Energy deposited beyond this level into the sample exerts no further influence on the demagnetization, but the traversing XUV radiation still generates an XMCD-SAS signal as long as the magnetization is nonzero.

The simulation results are shown as solid lines in Fig. 2. Shaded areas represent the uncertainty of the fit, which is predominantly due to the accuracy with which the FEL pulse lengths are known [15]. Our model is able to reproduce the experimental data with good accuracy over 


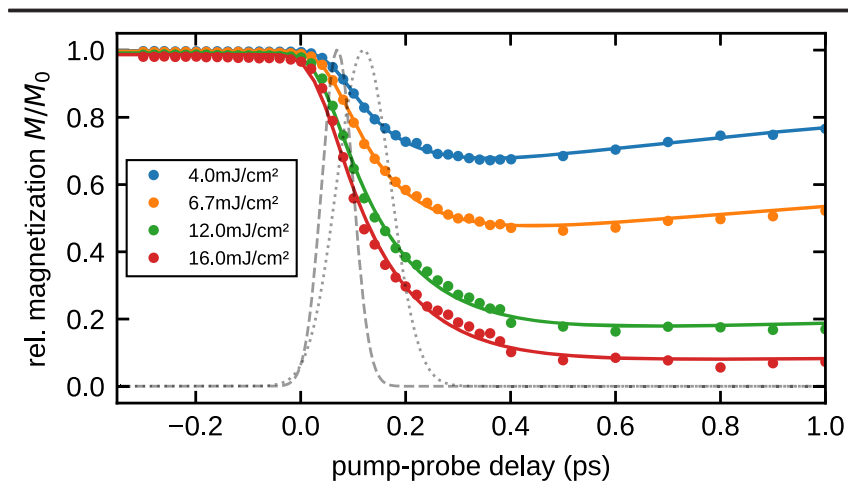

FIG. 3. Magnetization after an optical excitation measured by time-resolved MOKE on the same sample as used in the XUV experiment. The measurement is performed on the sample substrate, i.e., next to a silicon-nitride membrane. Solid lines are double-exponential fits to the data that yield the demagnetization time constant of $\tau_{m}=(113 \pm 9)$ fs for all pump fluences. For comparison, the temporal pulse envelope of the single shots used in the FEL experiment are indicated as dashed and dotted lines (70 fs and $120 \mathrm{fs}$, respectively). Note that these lines do not reflect the optical pump and probe pulses of $50 \mathrm{fs}$ pulse duration used in the MOKE experiment.

the entire fluence range and for both FEL pulse durations simultaneously. The fit yields a demagnetization time constant of $\tau_{m}=(100 \pm 20)$ fs. The saturation energy density is $F_{\text {sat }}^{\text {absorb }}=(20 \pm 5) \mathrm{kJ} / \mathrm{cm}^{3}$, which, given the absorption in a $1 \mathrm{~nm}$ slice of the sample material, corresponds to an incident fluence of $F_{\text {sat }}^{\text {incident }}=(22 \pm 5) \mathrm{mJ} / \mathrm{cm}^{2}$.

We perform time-resolved magneto-optical Kerr-effect (MOKE) measurements on the same samples investigated in the FEL experiment in order to independently determine the demagnetization dynamics as probed by this standard optical method. In contrast to demagnetization time constants, we do not expect fluence values to be quantitatively comparable between XUV and optical experiments. This is due to the vastly different photon energies and experimental setups used [15]. Here, we use 50 fs FWHM pulses of 800 and $400 \mathrm{~nm}$ wavelengths for the pump and probe beam, respectively. The MOKE measurements for different incident fluences are presented in Fig. 3. Given the presence of remagnetization on the picosecond timescale, the MOKE transients are fitted with a double-exponential fit taking demagnetization and remagnetization into account. This yields a demagnetization time constant of $\tau_{m}=$ $(113 \pm 9)$ fs for all fluences, with a negligible dependence on the demagnetization levels reached. The demagnetization time constants obtained via MOKE and the XUV single-shot experiments are thus in excellent agreement within the error, indicating that our demagnetization-based model is consistent with optically accessible ultrafast magnetization dynamics.

However, demagnetization rates for the XUV and nearoptical spectral ranges need not be exactly the same given that the initial electronic excitation triggering demagnetization is obviously different. The initial $3 p$ core vacancies generated by XUV excitation have a lifetime of 0.26 fs [27]. Their decay is dominated by rapid Auger processes, generating a population of excited secondary electrons above the Fermi level $\left(E_{F}\right)$. Furthermore, a sizeable fraction of the total absorption cross-section at the Co $M$ edges is due to photoexcitation of valence electrons. Given the large phase space accessible for electron-electron and electron-phonon scattering, even electrons several tens of eV above $E_{F}$ can be expected to loose their energy to within a few $\mathrm{eV}$ above $E_{F}$ on a sub-100 fs timescale. We thus expect the evolution to a hot but nonthermal electron distribution, i.e., to a situation comparable directly after excitation with $800 \mathrm{~nm}$, to proceed on a timescale shorter than our XUV pulse duration. While altered rates for electron-phonon spinflip scattering and spin-dependent inelastic scattering may be effective during this transition time, we do not expect a significant impact of these effects on the demagnetization rates at the temporal resolution of our experiment.

At fluences exceeding $2000 \mathrm{~mJ} / \mathrm{cm}^{2} /$ pulse, we observe small deviations of the experimental values compared to the model. For the longer $(120 \pm 20)$ fs pulses, the model seems to slightly overestimate the quenching of the XMCD-SAS cross-section, while the opposite is observed for the shorter $(70 \pm 20)$ fs FEL pulses. This might be due to simplifications of our model such as the strictly Gaussian temporal envelope of the XUV pulse. However, since we fit our model simultaneously to the experimental data for both pulse durations, it might also indicate that an additional effect, such as stimulated emission, starts to contribute to the signal quenching for the shorter pulses. Given the difference to the demagnetization model prediction, we estimate the contribution of any additional effect to the total XMCD-SAS quenching observed to be at most $10 \%$ at the highest fluences measured.

In contrast to the situation encountered here at the $M$ edge, stimulated emission has been reported to dominate the scattering and transmission process of $50 \mathrm{fs}$ FEL pulses in resonance with transitions from the Co $2 \mathrm{p}_{3 / 2}$ core level [6]. Following this, 25 and 2.5 fs single-spike FEL pulses have been used to investigate the fluence dependence at the Co $L_{3}$ absorption resonance [8]. In line with our reasoning, this experiment has shown a stronger effect for the shorter pulse lengths, corroborating the dominantly coherent nature of the XMCD-SAS fluence dependence at the Co $L_{3}$ edge. Recently, Higley et al. have shown that ultrafast demagnetization plays a nonnegligible role at the Co $L_{3}$ edge when monitoring x-ray absorption using 40 fs x-ray pulses [10]. This illustrates the competition of different mechanisms in the quenching of XMCD-based signals as a function of fluence.

A key parameter for stimulated emission to be observed during a single pulse is the lifetime of a population-inverted state created versus the coherence time of the excitation pulse [8]. In the case of a core excitation at the $M$ or $L$ edge, 
the limiting parameter is the respective core-hole lifetime. While both cases are dominated by Auger decay rates rather than spontaneous fluorescent decay, Co $3 p_{3 / 2,1 / 2}$ vacancies decay very fast via super Coster-Kronig transitions involving $3 \mathrm{~d}$ electrons, as reflected in the respective lifetime broadening $\Gamma_{M 3,2}$. For Co, the $2 \mathrm{p}_{3 / 2}$ lifetime is $1.4 \mathrm{fs}\left(\Gamma_{L 3}=0.47 \mathrm{eV}\right)$, while the $3 \mathrm{p}_{3 / 2}$ and $3 \mathrm{p}_{1 / 2}$ lifetimes are 0.26 fs $\left(\Gamma_{M 3,2}=2.5 \mathrm{eV}\right)$ [27]. Given that the Co $3 p$ lifetimes are more than five times smaller than the Co $2 p$ lifetimes, it is not surprising that coherent effects can be observed with sufficiently short pulses at the Co $L$ edge, especially when a pulse duration close to the core-hole lifetime can be employed as in Ref. [8]. Likewise, for pulse durations on the order of the demagnetization time, a significant contribution of ultrafast demagnetization to the fluence dependence of scattering should be expected also at the $L$ edges, as shown in Ref. [10].

In summary, we report for the first time the detailed fluence dependence of magnetic small-angle scattering from ferromagnetic domains in resonance with excitation of $3 p$ electrons. The data allow for quantitative comparison with theoretical models to explain the loss of scattering signal with fluence. Consistent with optical measurements, our observations for XUV pulse durations of 120 and $70 \mathrm{fs}$ can be explained taking into account only self-induced demagnetization. Given the $3 p_{3 / 2,1 / 2}$ core-hole lifetimes of the $3 \mathrm{~d}$ transition metals, we expect stimulated emission to play a significant role when moving on to intense fewfemtosecond or even attosecond pulses, which are becoming increasingly available.

[1] T. E. Glover, D. M. Fritz, M. Cammarata, T. K. Allison, S. Coh, J. M. Feldkamp, H. T. Lemke, D. Zhu, Y. Feng, R. N. Coffee, M. Fuchs, S. Ghimire, J. Chen, S. Shwartz, D. A. Reis, S. E. Harris, and J. B. Hastings, Nature (London) 488, 603 (2012).

[2] F. Bencivenga, R. Cucini, F. Capotondi, A. Battistoni, R. Mincigrucci, E. Giangrisostomi, A. Gessini, M. Manfredda, I. P. Nikolov, E. Pedersoli, E. Principi, C. Svetina, P. Parisse, F. Casolari, M. B. Danailov, M. Kiskinova, and C. Masciovecchio, Nature (London) 520, 205 (2015).

[3] M. Beye, S. Schreck, F. Sorgenfrei, C. Trabant, N. Pontius, C. Schüßler-Langeheine, W. Wurth, and A. Föhlisch, Nature (London) 501, 191 (2013).

[4] N. Rohringer, D. Ryan, R. A. London, M. Purvis, F. Albert, J. Dunn, J. D. Bozek, C. Bostedt, A. Graf, R. Hill, S. P. HauRiege, and J. J. Rocca, Nature (London) 481, 488 (2012).

[5] T. Wang et al., Phys. Rev. Lett. 108, 267403 (2012).

[6] B. Wu, T. Wang, C. E. Graves, D. Zhu, W. F. Schlotter, J. J. Turner, O. Hellwig, Z. Chen, H. A. Dürr, A. Scherz, and J. Stöhr, Phys. Rev. Lett. 117, 027401 (2016).

[7] J. Stöhr and A. Scherz, Phys. Rev. Lett. 115, 107402 (2015).

[8] Z. Chen, D. J. Higley, M. Beye, M. Hantschmann, V. Mehta, O. Hellwig, A. Mitra, S. Bonetti, M. Bucher,
S. Carron, T. Chase, E. Jal, R. Kukreja, T. Liu, A. H. Reid, G. L. Dakovski, A. Föhlisch, W. F. Schlotter, H. A. Dürr, and J. Stöhr, Phys. Rev. Lett. 121, 137403 (2018).

[9] L. Müller et al., Phys. Rev. Lett. 110, 234801 (2013).

[10] D. J. Higley, A. H. Reid, Z. Chen, L. L. Guyader, O. Hellwig, A. A. Lutman, T. Liu, P. Shafer, T. Chase, G. L. Dakovski, A. Mitra, E. Yuan, J. Schlappa, H. A. Dürr, W. F. Schlotter, and J. Stöhr, Nat. Commun. 10, 5289 (2019).

[11] F. Capotondi, E. Pedersoli, F. Bencivenga, M. Manfredda, N. Mahne, L. Raimondi, C. Svetina, M. Zangrando, A. Demidovich, I. Nikolov, M. B. Danailov, C. Masciovecchio, and M. Kiskinova, J. Synchrotron Radiat. 22, 544 (2015).

[12] E. Allaria et al., Nat. Photonics 7, 913 (2013).

[13] J. B. Kortright, J. Electron Spectrosc. Relat. Phenom. 189, 178 (2013).

[14] M. Schneider, C. M. Günther, C. von Korff Schmising, B. Pfau, and S. Eisebitt, Opt. Express 24, 13091 (2016).

[15] See Supplemental Material, which includes Ref. [16-20], at http://link.aps.org/supplemental/10.1103/PhysRevLett.125 .127201 for the spatial fluence distribution in the sample plane (section S.1); for the definition of the nominal fluence and calculation of error bars (section S.2); for details on data normalization (section S.3); for a quantitative analysis of the pulse-length dependent data separation (section S.4); for a detailed description of the demagnetization model (section S.5); for an illustration of the self-induced demagnetization at different pulse energies (Fig. S4); for the expected pulselength dependence of stimulated emission (section S.6); for the calculation of FEL pulse durations (section S.7); for a more exhaustive argument on comparability of fluence values (section S.8).

[16] J. Chalupský et al., Opt. Express 15, 6036 (2007).

[17] C. Li and P. Tam, Pattern Recogn. Lett. 19, 771 (1998).

[18] P. Finetti et al., Phys. Rev. X 7, 021043 (2017).

[19] F. Willems, S. Sharma, C. v. Korff Schmising, J. K. Dewhurst, L. Salemi, D. Schick, P. Hessing, C. Strüber, W. D. Engel, and S. Eisebitt, Phys. Rev. Lett. 122, 217202 (2019).

[20] B. Henke, E. Gullikson, and J. Davis, At. Data Nucl. Data Tables 54, 181 (1993).

[21] M. Schneider, C. M. Günther, B. Pfau, F. Capotondi, M. Manfredda, M. Zangrando, N. Mahne, L. Raimondi, E. Pedersoli, D. Naumenko, and S. Eisebitt, Nat. Commun. 9, 214 (2018).

[22] B. Keitel, E. Plönjes, S. Kreis, M. Kuhlmann, K. Tiedtke, T. Mey, B. Schäfer, and K. Mann, J. Synchrotron Radiat. 23, 43 (2016).

[23] B. Koopmans, G. Malinowski, F. Dalla Longa, D. Steiauf, M. Fähnle, T. Roth, M. Cinchetti, and M. Aeschlimann, Nat. Mater. 9, 259 (2010).

[24] B. Vodungbo et al., Sci. Rep. 6, 18970 (2016).

[25] S. Bonetti, M. C. Hoffmann, M.-J. Sher, Z. Chen, S.-H. Yang, M. G. Samant, S. S. P. Parkin, and H. A. Dürr, Phys. Rev. Lett. 117, 087205 (2016).

[26] J.-Y. Bigot, M. Vomir, and E. Beaurepaire, Nat. Phys. 5, 515 (2009).

[27] J. Campbell and T. Papp, At. Data Nucl. Data Tables 77, 1 (2001). 